\title{
Management of Garré's sclerosing osteomyelitis by endodontic therapy: A case report
}

\author{
Himanshu Aeran ${ }^{1, *}$, Avantika Tuli ${ }^{2}$, Ayush Pokhriyal ${ }^{3}$, Abhinav Chaudhary ${ }^{4}$ \\ ${ }^{1}$ Director Principal, Professor and Head, ${ }^{2}$ Professor, ${ }^{3,4}$ Post Graduate Student, ${ }^{1}$ Dept. of Prosthodontics \& Oral Implantology, ${ }^{2-}$ \\ ${ }^{4}$ Dept. of Pedodontics and Preventive Dentistry, Seema Dental College and Hospital, Veerbhadra Road, Rishikesh, Uttarakhand, \\ India
}

*Corresponding Author

Email: drhimanu@yahoo.com

\begin{abstract}
Garre's osteomyelitis is a distinctive type of chronic osteomyelitis associated with gross thickening of the periosteum of the bones and peripheral reactive bone formation resulting from mild irritation or infections. The condition is seen in children or young adults and the mandible is more affected than the maxilla in most cases. The following is a case report of a 10 year old boy having Garre's osteomylitis of the mandible due to the long standing infection and improper treatment of a carious tooth.
\end{abstract}

Keywords: Primary chronic osteomyelitis (PCO), Acute osteomyelitis (AO), Chronic osteomyelitis with peripheral periostites COPP.

\section{Introduction}

The word "osteomyelitis" originates from the ancient Greek words osteon (bone) and muelinos (marrow) and means infection of medullary portion of the bone. "Garrè's osteomyelitis is also known as periostitis ossificans, chronic nonsuppurative osteomyelitis of Garrè, Garrè's proliferative periostitis, chronic sclerosing inflammation of the jaw, chronic osteomyelitis with proliferative periostitis. Carl Alois Philipp Garré was a Swiss surgeon and bacteriologist who in 1893 published an article dealing with the manifestations of osteomyelitis. His name became associated with the disease, which became known as Garré's sclerosing osteomyelitis.

Garre's sclerosing osteomyelitis is a specific type of chronic osteomyelitis that primarily affects children and adolescents. ${ }^{1}$ Garré's osteomyelitis is a rare inflammatory disease of chronic nature, characterized by periosteal reactions. In the twenty-first century osteomyelitis presents as a sub-chronic condition and is more commonly associated with debilitated, immunosuppressed or medically compromised patient. ${ }^{2}$

Chronic osteomyelitis with peripheral periostites (COPP) commonly occurs in young patients with a mean age of 13 years. Sporadic cases have been reported in patients in their 20 s and in infants as young as 2 years. COPP is a non-suppurative inflammatory process. The most common provoking factors in the jaw region are a previous tooth extraction, tooth eruption, dental caries with associated periapical inflammation, periodontal infections, fractures and nonodontogenic infections. Most cases arise in the molar/premolar area of the mandible. Clinically, patients may present with a hard swelling of the jaw and facial asymmetry caused by this reactive process. Radiographic examinations typically show bony laminations parallel to each other and to the cortical surface of the involved bone. Areas of small sequestra or osteolytic radiolucencies can be found within the new bone. Appropriate radiographic angulation can highlight a radiolucent zone of soft tissue between the original bony cortex and the newly-formed reactive bone.

COPP presents a special radiographic feature, especially evident in computed tomography (CT) with three dimensional reconstruction, with new periosteal proliferation located in successive layers parallel to the condensed cortical bone. ${ }^{3}$ This phenomenon is also correlated with typical radiographic features such as an "onion peel" appearance. Other lesions that must be considered in differential diagnosis of COPP are Ewing's sarcoma, fibrous dysplasia, osteogenic sarcoma, infantile cortical hyperostosis, callus, exostosis, calcifying hematoma, and osteotomas. ${ }^{3}$

\section{Case Report}

A 10-year-old boy, reported to the Department of Paedontic and Preventive Denistry, Seema Dental College and Hospital, Rishikesh with the chief complaint of swelling over the lower jaw on the right side that was slowly increasing since last one month. Extraoral examination revealed that the swelling and was bony hard and did not show any mobility. (Fig. 1) There was slight pain on palpation of the swelling. There was an absence of any sinus tract, but a fixed indurated swelling presented on buccal vestibule of tooth \#46.

The patient's dental history included pain due to a carious tooth which was restored by a local dentist 2 months ago. 

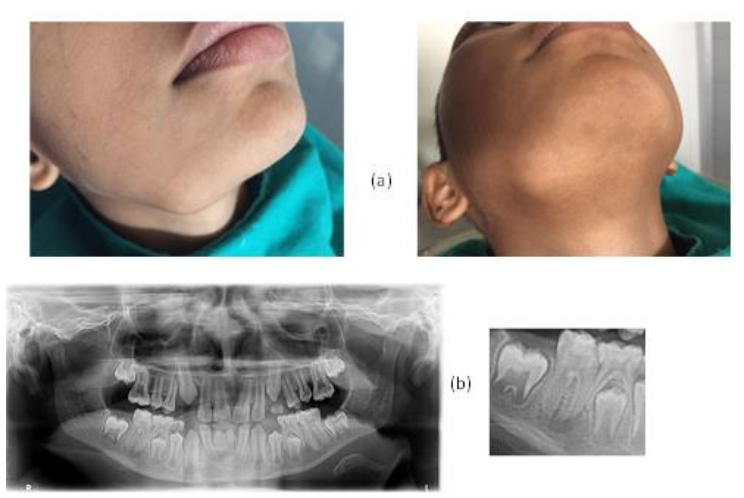

Fig. 1(a): Shows bony swelling at the lower border of mandible (b:) Shows OPG and IOPA showing onion peel apperance wrt 46

A periapical radiograph taken at the evaluation appointment showed apical periodontitis associated with tooth \#46 (Fig. 2). An OPG was done which showed an onion-peel appearance of the cortical plate in the area of tooth \#46, consistent with proliferative periostitis. A diagnosis of pulpal necrosis and chronic apical periodontitis associated with proliferative periostitis was made w.r.t 46. The patient's lower right quadrant was anesthetized, and tooth \#46 was isolated and first faulty restoration was removed and acess cavity preaparation revealed necrotic pulp with no apparent odor or purulence present.

The root canal was irrigated between instrumentation with $2.5 \%$ sodium hypochlorite. The canal was dried, calcium hydroxide was placed in the canal, and a sterile cotton pellet was sealed in the pulp chamber with interim restoration (cavitG). At the next appointment 2 weeks later, the patient had an absence of pain on palpation and there was a reduction in the swelling on the right side of the mandible. Intracanal medicament was changed and followed by the interim restoration and patient was recalled after 3 months. On the next visit the hard bony swelling had completely disappeared (Fig. 2) and the endodontic treatment was completed using apexit plus as a roortcanal sealer. An OPG was taken for which raveled the total healing of the lesion with respect to \#46. (Fig. 3)
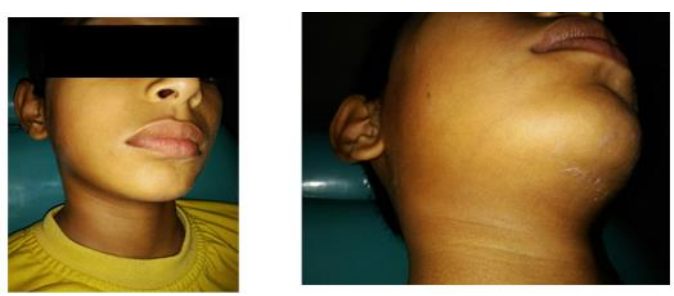

Fig. 2: Shows clinical picture of extraoral view of patient the hard bony swelling had completely disappeared

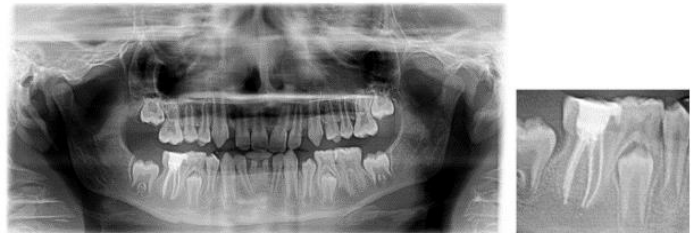

Fig. 3: The OPG raveled the total healing of the lesion with respect to \#46

\section{Discussion}

Osteolytic mandibular lesions are typically bulky, with irregular radiolucency including both expansion and destruction of the cortical plate. The treatment of patients with COPP aims to remove identifiable sources of inflammation. Usually, removal of the infected tooth and curettage of its socket are required to cure the lesion. In some cases, however, endodontic treatment has been reported as a successful means of treating odontogenic causes of proliferative periostitis.

The elimination of periapical infection was achieved by endodontic therapy and the complete bone remodeling was seen radiographically after three months follow-up of a case that was diagnosed as garres osteomylitis. ${ }^{6}$

Zand $\mathrm{V}$ et al support the use of intercanal medicament (calcium hydroxide) for the treatment of osteomilitis. ${ }^{6}$

This disease is usually found in younger age group, so whenever the tooth is restorable root canal treatment with follow up should be considered as a main treatment option. In young patients with Garre's osteomyelitis the non-surgical endodontic treatment should be considered as a treatment option rather than extraction. $^{7}$

Clinically, this reactive process accounts for the hard swelling of the jaw and the subsequent facial asymmetry with which patients may present. The lesion is usually asymptomatic with no accompanying general and local signs of inflammation, although the clinical picture may vary widely. Garre's osteomyelitis presents a characteristic radiographic feature, especially in occlusal radiograph showing new periosteal proliferation located in successive layers to the condensed cortical bone. This is the typical radiographic feature of Garre's osteomyelitis and is well known as "onion skin" appearance. ${ }^{8}$

Antibiotic therapy alone is ineffective, independent of the administration route, because the "bone sequestration" found in the chronic disease comprises fragments of necrotic bone and thus does not present a blood supply that would allow antibiotic to arrive at the infected tissue. ${ }^{9}$

Chronic osteomyelitis presents low mortality but high morbidity. Clinically, the chronic phase is always preceded by an acute infectious process, with phlogistic signs (pain, heat, redness, tumor growth, deformity and limitation). However, in patients who have become debilitated through other diseases, as was the case in 
our patient, the initial condition may be masked, which has the consequence that the diagnosis will only become possible in the chronic phase. ${ }^{10}$

Suma $\mathrm{R}$ et al advocate the use of metronidazole and gentamicin as an irrigating system for after the systemic use of analgesic and antibiotic. ${ }^{10}$

\section{Conclusion}

Garres osteomyelitis is a type of chronic osteomyelitis with subperiostel thickening. It is associated with low grade infection, which may be due to dental caries. Clinically it appears as asymmetrical swelling. Radiographically, it has onion peel appearance which confirms it as Garres osteomyelitis. Elimination of pulpal and associated periapical infection through routine endodontic therapy is observed to be effective in allowing resolution of the local periostitis of Garre's osteomyelitis. It has different treatment modalities. It can be treated with systemic antibiotic therapy and irrigation with gentamicin. It can also be treated with calcium hydroxide dressings which has better results without any antibiotic therapy

\section{References}

1. Oulis C, Berdousis E, Vadiakas G, Goumenos G. Garres osteomyelitis of an unusual origin in a 8 year old child: A case report. Int J Pediatr Dent 2000;10:240-4.

2. Grayson M L, Gibbons G W, Balogh K, Levin E, Karchmer A W. Probing to bone in infected pedal ulcers. A clinical sign of underlying osteomyelitis in diabetic patients. JAMA 1995;273:721-723.

3. Yen-Ching Chang, Yi-Shing Shieh, Shiao-Pieng Lee, YiJan Hsia, Chih-Kung Lin, Shin Nieh, Huey-Kang Sytwu, Yuan-Wu Chen. Chronic osteomyelitis with proliferative periostitis in the lower jaw. Journal of Dental Sciences (2015);10:450-455.

4. Carnelio S, Pai K, Rao N, Solomon M, Ahasan A. Metastatic osteosarcoma to the maxilla: a case report and a review of the literature. Quintessence Int 2002;33:3979.

5. Angiero F, Vinci R, Sidoni A, Stefani M. Mesenchymal chondrosarcoma of the left coronoid process: report of a unique case with clinical, histopathologic, and immunohistochemical findings, and a review of the literature. Quintessence Int 2007;38:349-55.

6. Vahid Zand, DMD, MSc, Mehrdad Lotfi, DMD, MSc, and Sepideh Vosoughhosseini, DMD, MScProliferative Periostitis: A Case Report JOE 2008;34(4):481-83.

7. Jayasenthil A, Venkatalakshmi A, Balagopal S. Nonsurgical Endodontic Management of Garre's Osteomyelitis: A Case Report. BJMMR;2015:9(3):1-4.

8. Eswar N. Garres Osteomyelitis: A case report. J Indian Soc Pedo Prev Dent 2001;19:157-9

9. Vienne P., Exner G.U. Garrè sclerosing osteomyelitis. Orthopade.1997;26(10):902-907.

10. Suma R., Vinay C., Shashikanth M.C., Subba Reddy V.V. Garrè's sclerosing osteomyelitis. J Indian Soc Pedod Prev Dent. 2007;25(Suppl.):S30-S33. 